\title{
DEVELOPING OPEN TRAVEL ALLIANCE-BASED ONTOLOGY OF GOLF
}

\author{
Agnieszka Cieslik \\ Dep. of Mathematics and Information Technology, Warsaw University of Technology, Warsaw, Poland \\ agnieszka.cieslik@gmail.com \\ Maria Ganzha, Marcin Paprzycki \\ Systems Research Institute Polish Academy of Science, Warsaw, Poland \\ maria.ganzha,marcin.paprzycki@ibspan.waw.pl
}

Keywords: OTA, ontology, golf, agent-based system, travel support system

\begin{abstract}
For usage of ontologies to become more prevalent, not only new ontologies have to be created to represent the world, but also ontological support for existing domain-specific real-world standards has to be provided. One of such standards that gains popularity is the Open Travel Alliance (OTA) messaging system that defines, among others, the way that entities should communicate about golf as a travel-related entity. The aim of this paper is to outline our efforts leading toward creating an ontology of golf that would match the OTA messaging specification.
\end{abstract}

\section{INTRODUCTION}

Our current work is focused on developing an agentbased system that provides comprehensive support for needs of a traveler. To this effect we have been involved in two related projects. First project is the design and implementation of a model agent-based ecommerce system (see (Bădică et al., 2007b; Bădică et al., 2007a), and references to our earlier work contained there). This project is focused on utilization of price negotiations in a comprehensive e-commerce scenario, and was extended to facilitate possibility of airline ticket auctioning (Vukmirovic et al., 2006c; Vukmirovic et al., 2006a; Vukmirovic et al., 2006b; Szymczak et al., ; Vukmirovic et al., 2007). Second, we work on creation of an agent based Travel Support System (TSS) (Salam and Stevens, 2006; Gawinecki et al., 2005b; Gordon and Paprzycki, 2005). In the $T S S$, travelers are going to find, among others, personalized information about restaurants, hotels, historical points of interest, local weather etc. The main idea of the TSS is to utilize a central repository of semantically demarcated travel data, and use it to facilitate personalized information provisioning (Gawinecki et al., 2005b; Gawinecki et al., 2005c).

These two projects (airline ticket auctioning system and Travel Support System) were developed separately. It was only in (Vukmirovic et al., 2006c) where we have looked into issues involved in their possible merger. The key consideration was related to the fact that ontologically demarcated data is the central component of the TSS (Salam and Stevens, 2006; Gawinecki et al., 2005b; Gordon and Paprzycki, 2005). Therefore, the two projects were conceptually merged through development of a common travel ontology. First, still within the TSS, we have developed and then merged ontologies of hotels and restaurants. These two ontologies were developed utilizing a very pragmatic approach to ontology building and were based on the concept of a hotel as represented in travelrelated WWW sites and concept of a restaurant as proposed in the ChefMoz project, respectively (Gawinecki et al., 2005a; Gordon et al., 2005).

In the airline ticket auctioning system we have pursued an even more reality-grounded approach. Here, of particular importance is the fact that all air-travel related activities are regulated by IATA, the global air-travel governing organization. This fact is not often recognized by non-practitioners and as a result, as shown in (Vukmirovic et al., 2006c; Vukmirovic et al., 2006a), most of existing air travel ontologies, being typically developed as an academic exercise, lack specific features that are required by IATA as well as a number of additional features that are needed in day-to-day operation of existing airlines. Separately, the Open Travel Alliance (OTA) (OTA, a) 
Table 1: Summary of OTA golf messages

\begin{tabular}{|c|c|}
\hline Message type & List of fields \\
\hline $\begin{array}{l}\text { OTA_GolfCourseSearchRQ_-request for course information; } \\
\text { used to find golf courses that satisfy a given set of criteria }\end{array}$ & $\begin{array}{l}\text { Architect, ADAChallenged, Slope, Metal Spikes, Caddies available, Yardage, } \\
\text { Personal Carts Permitted, Grass Type, Singles Confirmed }\end{array}$ \\
\hline $\begin{array}{l}\text { OTA_GolfCourseSearchRS-list of courses that meet the re- } \\
\text { quested criteria; if attribute is specified as Required (set to Yes) } \\
\text { then only courses that meet criteria will be returned; if Required } \\
\text { attribute is No a course that does not meet a given criteria may } \\
\text { be included in the list }\end{array}$ & $\begin{array}{l}\text { Golf Course ID, Golf Course address, Contact information-telephone number, } \\
\text { List of requested criteria }\end{array}$ \\
\hline $\begin{array}{l}\text { OTA_GolfCourseAvailRQ - requests information about avail- } \\
\text { ability of a specific golf course }\end{array}$ & $\begin{array}{l}\text { Golf Course ID, Tee Time-start and end date, Number of golfers, Number of } \\
\text { holes, Maximum price for one person }\end{array}$ \\
\hline $\begin{array}{l}\text { OTA_GolfCourseAvailRS_-provides information about field } \\
\text { availability }\end{array}$ & $\begin{array}{l}\text { Golf Course ID, Tee Time, Number of golfers, Number of holes, Maximum price } \\
\text { for one person, List of fees. Fee has name, information about amount, currency } \\
\text { and taxes }\end{array}$ \\
\hline $\begin{array}{l}\text { OTA_GolfCourseResR } Q \text {-requests a reservation of a given golf } \\
\text { course }\end{array}$ & $\begin{array}{l}\text { Information about person who makes reservation (first and last name, address, } \\
\text { date of birth, telephone number), Mean of payment, Date of game, Number of } \\
\text { golfers, Number of carts, List of fees }\end{array}$ \\
\hline $\begin{array}{l}\text { OTA_GolfCourseResRS-confirms (or denies) reservation of a } \\
\text { given golf course }\end{array}$ & $\begin{array}{l}\text { Reservation ID, Information about person who makes reservation (first and last } \\
\text { name, address, date of birth, telephone number), Mean of payment (credit cart } \\
\text { information), Date of game, Number of golfers, Number of carts, List of fees, } \\
\text { Information concerning cancellation penalties and date and time by which a can- } \\
\text { cellation must be made }\end{array}$ \\
\hline
\end{tabular}

has proposed a set of messages that organizations involved in travel-related activities can use to meaningfully communicate about travel entities such as flights or golf course reservations. Interestingly, one can infer that OTA messages may be currently on the way to become an industry-wide standard. For instance, a number of travel-related businesses (e.g. some of US-based airlines) have accepted OTA messages in their business practices. Let us also note that, from the technical perspective, OTA messaging is an open standard that consists of a set of XML-demarcated messages. Obviously, by the very nature of their design and function, these messages represent a certain conceptualization of the "world of travel." However, they do not explicitly define an ontology. In (Vukmirovic et al., 2007) we have proposed how OTA airtravel-related messages can be used as a basis for development of an ontology of air-travel. Furthermore, we have utilized IATA manuals, and practical knowledge of a member of our team, in an attempt to assure compliance of the proposed ontology with airtravel regulations and practices. Finally, the proposed air-travel ontology was successfully merged with the ontology of restaurant and hotel. The results (complete ontology of restaurants, hotels and air travel) can be found at (tss, ). The aim of this paper is to describe how, in an attempt at extending the TSS ontology and functionality, the OTA golf messaging has been turned into an OTA ontology of golf.

To this effect we proceed as follows. In the next sec- tion we describe the OTA golf messages. We follow with the specification of concepts that have to be represented in the OTA golf ontology. Next we analyze which concepts should be re-used from the TSS ontology (in order to allow later merging of these ontologies). Finally we present the most important parts of the proposed OTA ontology of golf.

\section{OTA golf messages}

Let us now briefly describe golf-related OTA messages (description is based on (OTA, b)). As in the case of all OTA messages, they come in pairs. There is alway a request (RQ) message (a query) and, corresponding to it, a response (RS) message. For the golf-course-related communications the OTA standard identifies three pairs of messages summarized in Table 1. In short, these messages provide the following functionalities: (1) ability to find a golf course with specific characteristics, (2) check if a course of interest is available at a specific time and under a specific set of conditions (e.g. below a certain maximum price), and (3) make an actual reservation.

To illustrate the specific form that OTA messages take, in Figure 1 we present an example on an OTA_GolfCourseAvailRQ message (based on (OTA, b)). In this message two friends specify that they would like to play golf on October 31 st, and the re- 
quested tee-off time is to be between 1:00 and 2:30. They are interested in playing at a specific golf course with the identifier FL3421. The maximum price that they are willing to pay for 18 holes is $\$ 80.00$ per person. The aim of this message is to find if the FL3421 course is available at a given time and if the price condition is satisfied.

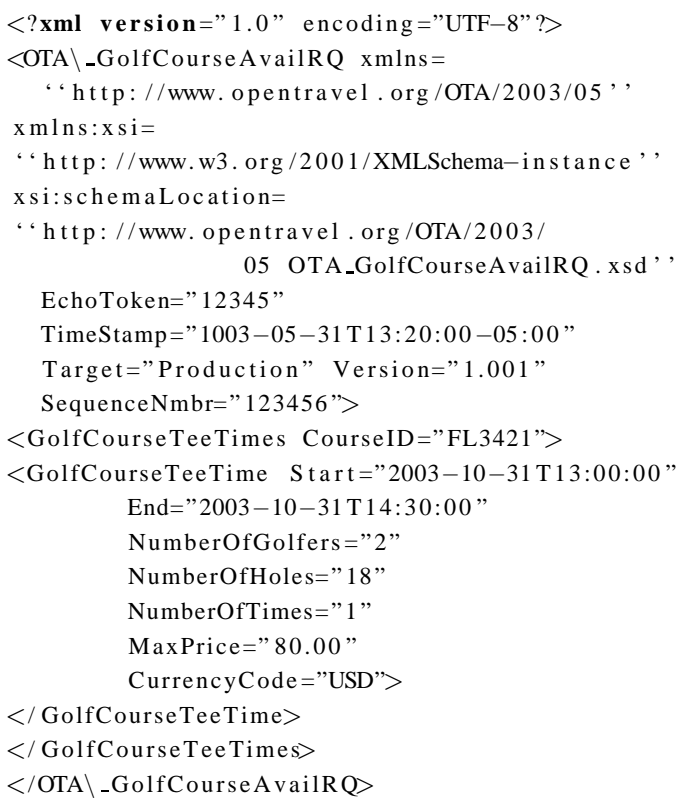

Figure 1: Example of an OTA golf course availability query message

In response to the OTA_GolfCourseAvailRQ message depicted in Figure 1, the OTA_GolfCourseAvailRS message presented in Figure 2 could have been received. This response indicates that the requested FL3421 course is available on October 31st, with the tee-time between 1:00 and 2:30. Furthermore, the green fee is $\$ 70.00$ per person, while the cart fee is $\$ 10.00$ per person.

As a result, a GolfCourseResR $Q$ message could be send, requesting a reservation at a specific time. This message would then have to be followed by a GolfCourseResRS message that would confirm the reservation.

\section{Designing the ontology-preliminary considerations}

Now, we can discuss how OTA golf-course-related messages can be used as a basis for the development

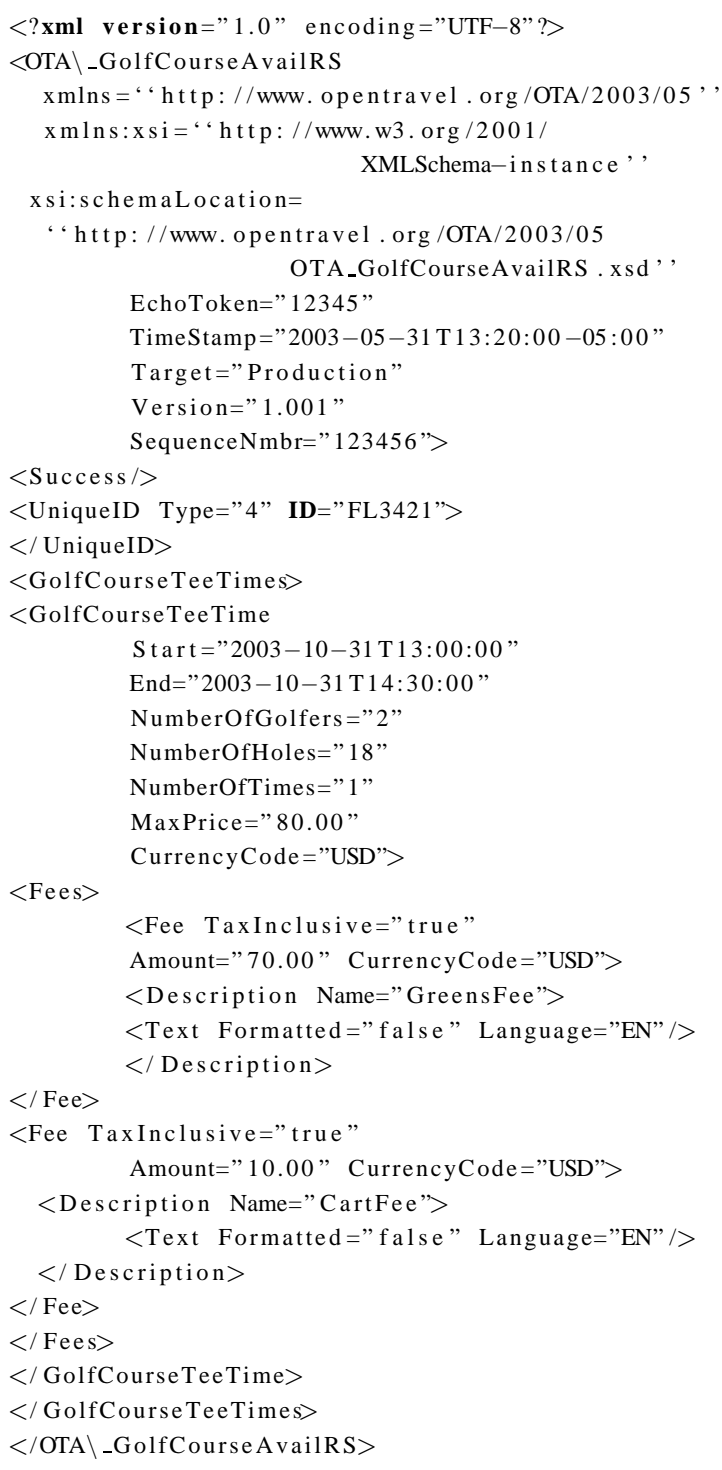

Figure 2: Example of an OTA golf course availability response message

of an OTA golf course ontology. Analysis of OTA golf related messages showed us a need for defining of two core concepts: Golf Course and Golf Course Tee Time. The first one is going to uniquely identity a given golf course and its features. This concept is related directly to the first of OTA message pairs, where a golf course with a specific set of features is sought. This concept is similar to the restaurant and hotel concepts from the TSS ontology of travel. All three concepts define a static object and a set of its features. The second concept will define all information that is necessary for completing a reservation of 
a golf course. Thus, the Golf Course Tee Time concept defines dynamic characteristics of a static object specified in the Golf Course object. The Golf Course concept and its features are represented in Table 3

Table 2: Golf Course concept and its features

\begin{tabular}{|l|l|}
\hline \multicolumn{2}{|l|}{ Class GolfCourse } \\
\hline Course ID & $\begin{array}{l}\text { ID originates from the OTA_GolfCourseSearchRS } \\
\text { message; can be used for getting information } \\
\text { about golf course availability and for making } \\
\text { reservations }\end{array}$ \\
\hline Address & Address of golf course \\
\hline Contact & Contact information (e.g. telephone number) \\
\hline Features & List of golf course features \\
\hline
\end{tabular}

In Table 3 we list features that constitute the necessary information to define the Golf Course Tee Time concept. Since the "names of features" are self-explanatory, we do not define them further. It is of value to compare the list of features defining the Golf Course Tee Time concept with the content of the golf course availability querying (GolfCourseAvailRQ) message presented above.

Table 3: Golf Course Tee Time concept and its features

\begin{tabular}{|l|}
\hline Class GolfCourseTeeTime \\
\hline Course ID \\
\hline Start date and time \\
\hline End date and time \\
\hline Price \\
\hline Max price for one person \\
\hline Number of holes \\
\hline Number of golfers \\
\hline Number of games \\
\hline List if fees \\
\hline
\end{tabular}

After identifying two concepts that are going to constitute the core of the OTA golf ontology, we have to address the following question: how does this ontology relate to the existing TSS ontology. In other words, we have to establish which already existing / defined concepts can (and should) be immediately reused in the new ontology.

\subsection{Common concepts with the TSS ontology}

Ontology re-use is one of important concepts in ontological engineering (Fensel, 2003). Therefore we should re-use as much as possible of the existing ontologies (and we have done so within the TSS ontology). Furthermore, the OTA golf course ontology, should be integrable with the TSS ontology and thus utilize immediately as many of its concepts as pos- sible. Thus we have identified existing concepts that could be re-used; let us list them here.

Outdoor Location-geographical location is one of common features of objects populating the TSS (restaurant, hotel, airport). Obviously, it is also a concept that is directly related to the golf course. The OutdoorLocation concept (class) describes geographical location through a set of geographical properties, such as: street address, country, city/town, region, zip code, reference points or location description (see the TSS ontology available at (tss, ) for a complete listing). In the TSS ontology, the Hotel, the Restaurant and the Airport classes are sub-classes of the OutdoorLocation class. Therefore, the proposed class GolfCourse should also become a subclass of OutdoorLocation. This is a natural decision as the Golf Course should be an object of the same "stature" as the other objects mentioned here.

Discounts - in general, is the class that contains the following information:

- code of the particular discount,

- amount of reduction of the base-price,

- short description of the discount policy.

However, when dealing with air travel support we have realized that IATA defined specific air travel discount codes. Therefore, the question has arisen: how to integrate these with hotel and restaurant discount codes (including both OTA-specific and general discounts-these omitted in the OTA specification). For the purpose of integration of ontologies, specific discounts codes were distinguished and defined as subclasses of the general DiscountTypes class. Specifically, in the TSS there exist three classes defining possible discounts:

- OTADiscountTypes-discount types originating from the OTA specification

- IATADiscountTypes - discount types originating from the IATA specification

- DiscountTypes - general class; all discount types

Obviously, classes OTADiscountTypes and IATADiscountTypes are subclasses of DiscountTypes. Note that since the proposed ontology of golf is based on OTA messages, the discount concepts used here belong to the OTADiscountTypes class.

The remaining common parts between the TSS and the OTA golf course ontologies are:

- MeanOfPayment-concept defining possible mean of payment (e.g cash, credit card, check, etc.)

- AdressRecord-class that in the TSS ontology describes the address 
- Currency-defines what is the currency that the fees are in

- FareTax-information about taxes

- Contacts-possible ways of contacting an entity (e.g. telephone number)

\section{The OTA golf ontology}

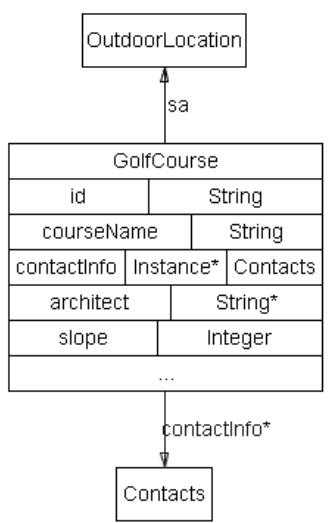

Figure 3: Golf Course concept; graphical representation

Based on the above considerations we can now briefly discuss the most important aspects of the proposed OTA golf ontology. Let us start from definitions of the two basic classes, the main class defining the concept of the Golf Course is presented in Figure 4 (in the form of the standard RDF notation) and in Figure 3 (in the visual representation).

As can be easily seen in the graphical representation, the GolfCourse class is a subclass of OutdoorLocation and utilizes the Contacts concept (from the TSS ontology). In its definition we use strings for: $i d$, courseName, architect; and an integer for the slope. The second concept that belongs to the core of the OTA golf course ontology is Golf Course Tee Time is presented in Figure 5 (in the RDF notation) and in Figure 6 in its graphical representation.

Observe that while the GolfCourseTeeTime class is relatively simple itself (it consists of strings for: startDate, endDate and golfCourseID; float for maxPrice; and an integer for numberOfTimes), it utilizes also a fairly extensive concept of a Fee. This points out to the fact that in addition to the two basic concepts (classes GolfCourse and GolfCourseTeeTime) we had to define the following additional concepts / classes:

- Price-concept of price (includes: amount, taxes, currency, etc.)
- Fee-concept of fee (e.g. green fee, cart fee)

- Description-additional descriptions that are needed for the traveler to be able to effectively utilize the information provided by the system

Note that the concept of the Price is similar to that used in the TSS ontology, however in the case of a golf course it is much less complicated than in the case of air travel. Therefore we have decided, for the time being, to leave this concept golf-specific and return to this issue when the OTA golf ontology is going to be integrated with the TSS ontology. At the same time, the Fee concept, which is the superclass of the Price, is golf-specific. Finally, in Figure 6, we can see how the DiscoutTypes concept (from the TSS ontology) is utilized. To complete the description of the proposed OTA golf ontology, in Figure 7 we depict the Price class (in the RDF notation), in Figure 8 we present the Fee class (in the RDF notation), while in Figure 9 we introduce the Description class (also in the RDF notation).

\section{Concluding remarks}

In this paper we have discussed how an OTA golf ontology can be reverse engineered from the OTA golf messaging system. In the proposed approach we have paid attention to re-use of concepts that have been defined earlier within the TSS ontologies of restaurant, hotel and air-travel. In this way the proposed OTA golf ontology can be easily integrated with the TSS ontology. The only issue that has to be solved is the utilization of the Price concept across ontologies of multiple travel entities (as some of them have more complicated price concept than others). This issue is being resolved currently as the OTA golf ontology is being merged with the TSS ontology and the results will shortly be available at (tss, ). Finally, let us note that we have already created a translator that allows us to deal with the following scenario. Let us assume that information about golf courses is stored in a repository (e.g. a Jena repository (jen, )) as instances of the above described OTA golf ontology. Such a repository has to be queried using one of languages designed for this purpose (e.g. SPARQL (SPA, )). At the same time, other entities "want to" communicate with our system using OTA messaging. This being the case OTA request messages have to be translated into SPARQL queries, while responses to SPARQL queries have to be translated back to OTA response messages. We have implemented the needed translator and will discuss its design in a separate report. 


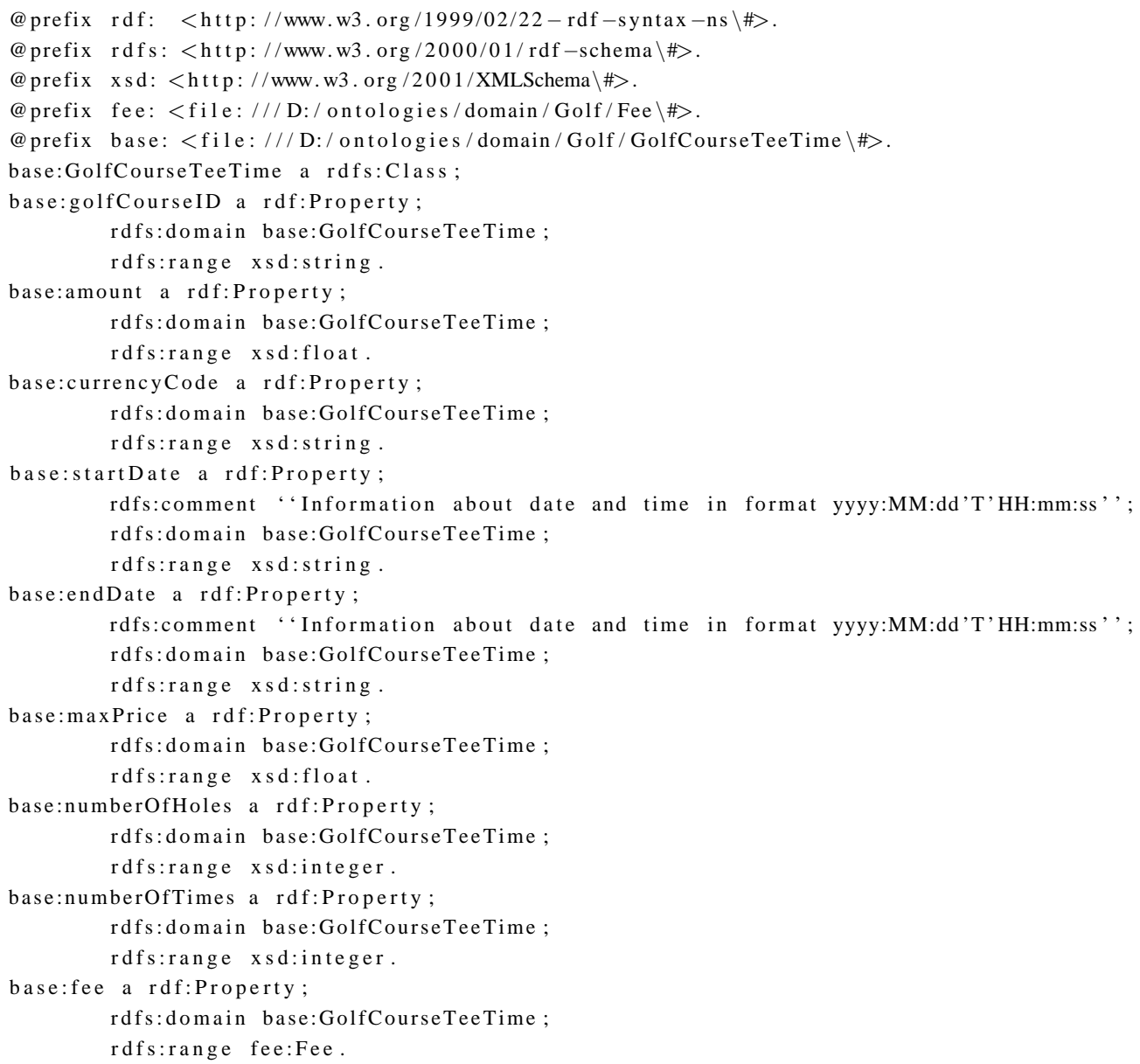

Figure 5: Golf Course Tee Time concept; proposed GolfCourseTeeTime class

\section{REFERENCES}

Jena-RDF persistency engine. http://jena.
sourceforge.net/.

OTA-Open Travel Alliance. http://www.opentravel. org/.

OTA golf messaging manual. OTA_ MessageUserGuide2006V1.0.

SPARQL-RDF query language. http://www.w3.org/ $\mathrm{TR} /$ rdf-sparql-query/.

Travel support system, software repositories. http: //www. e-travel.sourceforge.

Bădică, C., Bădită, A., Ganzha, M., and Paprzycki, M. (2007a). E-Service Intelligence-Methodologies, Technologies and Applications, chapter Developing a Model Agent-based E-commerce System, pages 555578. Springer, Berlin.

Bădică, C., Ganzha, M., and Paprzycki, M. (2007b). Joutnal of Universal Computer Science, volume 13, chapter Implementing Rule-Based Automated Price Nego- tiation in an Agent System, pages 244-266. Springer, Berlin.

Fensel, D. (2003). Ontologies: A Silver Bullet for Knowledge Management and Electronic Commerce. Springer-Verlag New York, Inc., Secaucus, NJ, USA.

Gawinecki, M., Gordon, M., Nguyen, N. T., Paprzycki, M., and Szymczak, M. (2005a). Rdf demarcated resources in an agent based travel support system. In et. al., M. G., editor, Informatics and Effectiveness of Systems, pages 303-310, Katowice. PTI Press.

Gawinecki, M., Gordon, M., Nguyen, N. T., Paprzycki, M., and Vetulani, Z. (2005b). chapter Ontologically Demarcated Resources in an Agent Based Travel Support System, pages 219-240. Advanced Knowledge International, Adelaide, Australia.

Gawinecki, M., Kruszyk, M., and Paprzycki, M. (2005c). Ontology-based stereotyping in a travel support system. In Proc. of the XXI Fall Meeting of Polish Information Processing Society, pages 73-85. PTI Press.

Gordon, M., Kowalski, A., Paprzycki, M., Pełech, T., Szymczak, M., and Wasowicz, T. (2005). Internet 


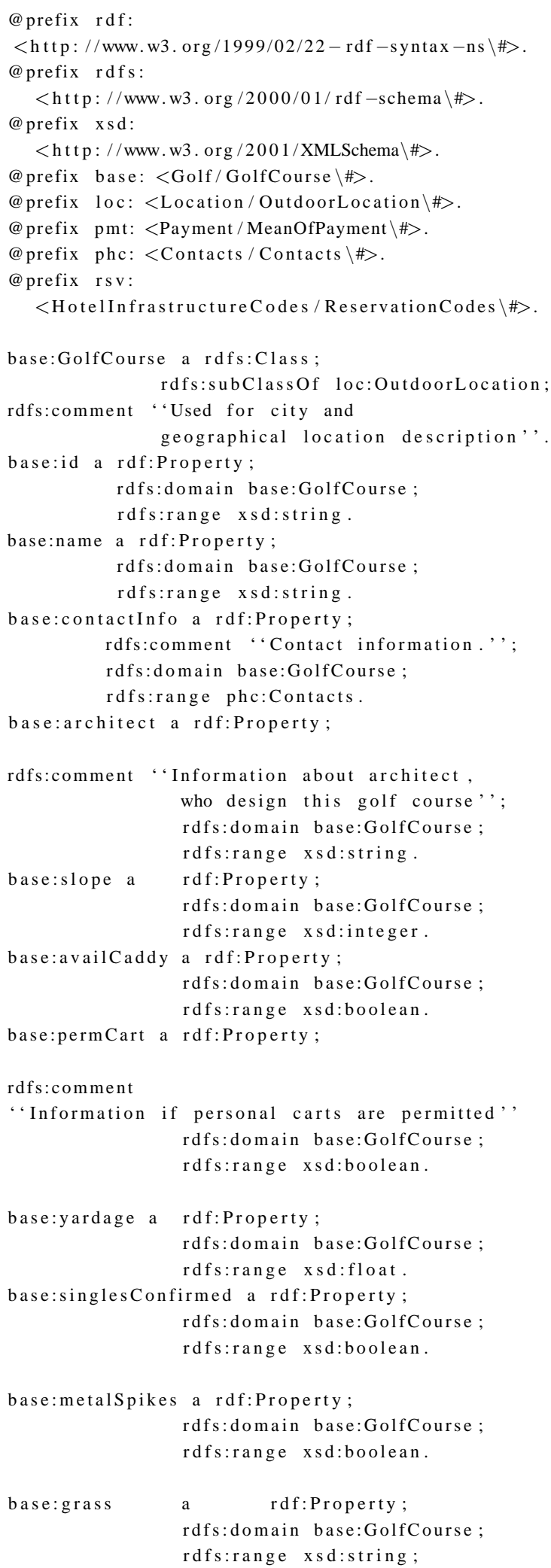

Figure 4: Golf Course concept; proposed GolfCourse class

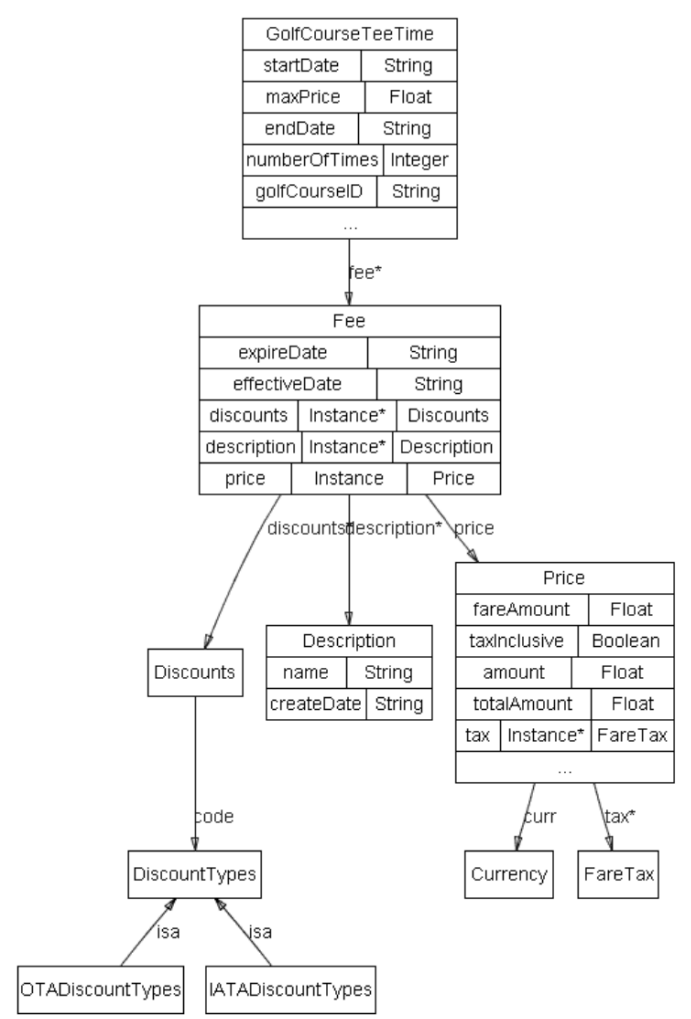

Figure 6: Golf Course Tee Time concept; graphical representation

2005, chapter Ontologies in a Travel Support System, pages 285-300. Technical University of Wroclaw Press.

Gordon, M. and Paprzycki, M. (2005). Designing agent based travel support system. In ISPDC'2005:Proc. of the ISPDC 2005 Conference, pages 207-214, Los Alamitos, CA. IEEE Computer Society Press.

Salam, A. F. and Stevens, J., editors (2006). chapter Utilizing Semantic Web and Software Agents in a Travel Support System, pages 325-359. Idea Publishing Group, Hershey, USA.

Szymczak, M., Gawinecki, M., Vukmirovic, M., and Paprzycki, M. Ontological Reusability in State-of-theart Semantic Languages, pages 129-142. PTI Press.

Vukmirovic, M., Ganzha, M., and Paprzycki, M. (2006a). Developing a Model Agent-based Airline Ticket Auctioning System, pages 297-306. Springer, Berlin.

Vukmirovic, M., Paprzycki, M., and Szymczak, M. (2006b). Designing ontology for the open travel alliance airline messaging specification. In et. al., M. B., editor, Proceedings of the 2006 Information Society Multiconference, pages 101-105. Josef Stefan Institute Press. 


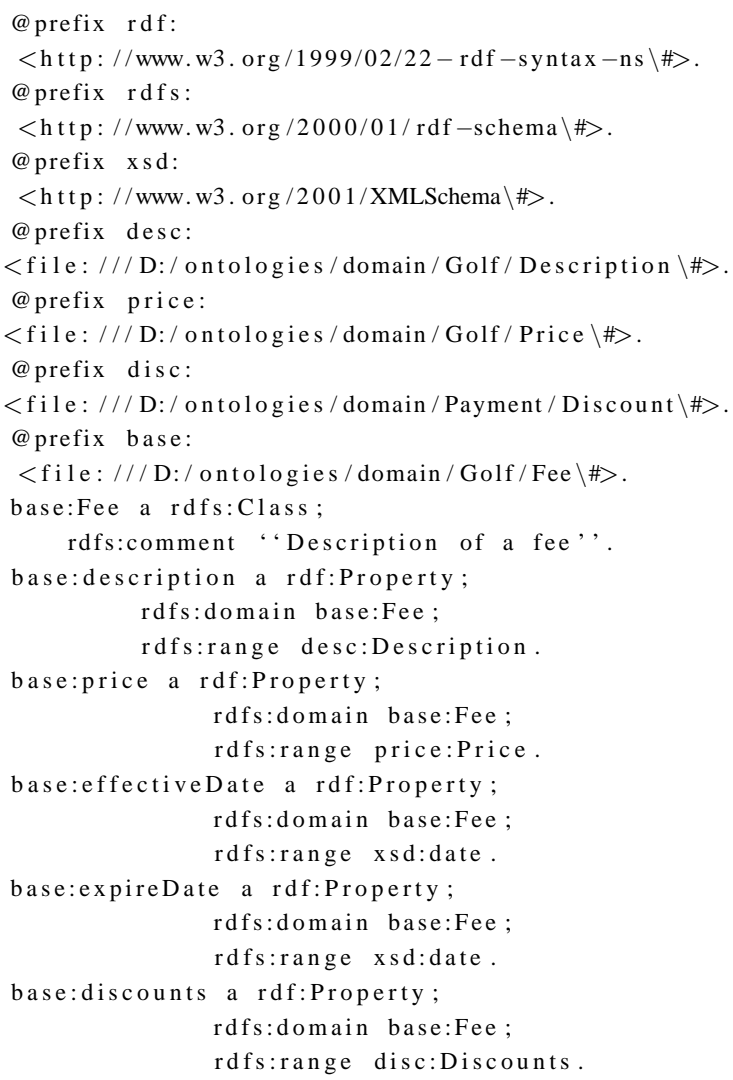

Figure 8: Fee concept; proposed Fee class

@ prefix rdf:

$<$ http: //www.w3. org/1999/02/22-rdf-syntax -ns $\backslash \#>$. @ prefix rdfs:

$<$ http: //www.w3.org/2000/01/rdf-schema \\#>.

@ prefix xsd:

$<$ http: //www.w3.org/2001/XMLSchema \\#>

@ prefix base:

<file:///D:/ontologies/domain/Golf/Description \\#>.

base:Description a $\mathrm{rdfs}: \mathrm{Class}$; rdfs:comment "Description".

base:name a rdf:Property

rdfs:domain base:Description

rdfsirange xsd:string.

base:createDate a rdf:Property;

rdfs:domain base:Description; rdfs:range xsd:date.

Figure 9: Description concept; proposed Description class

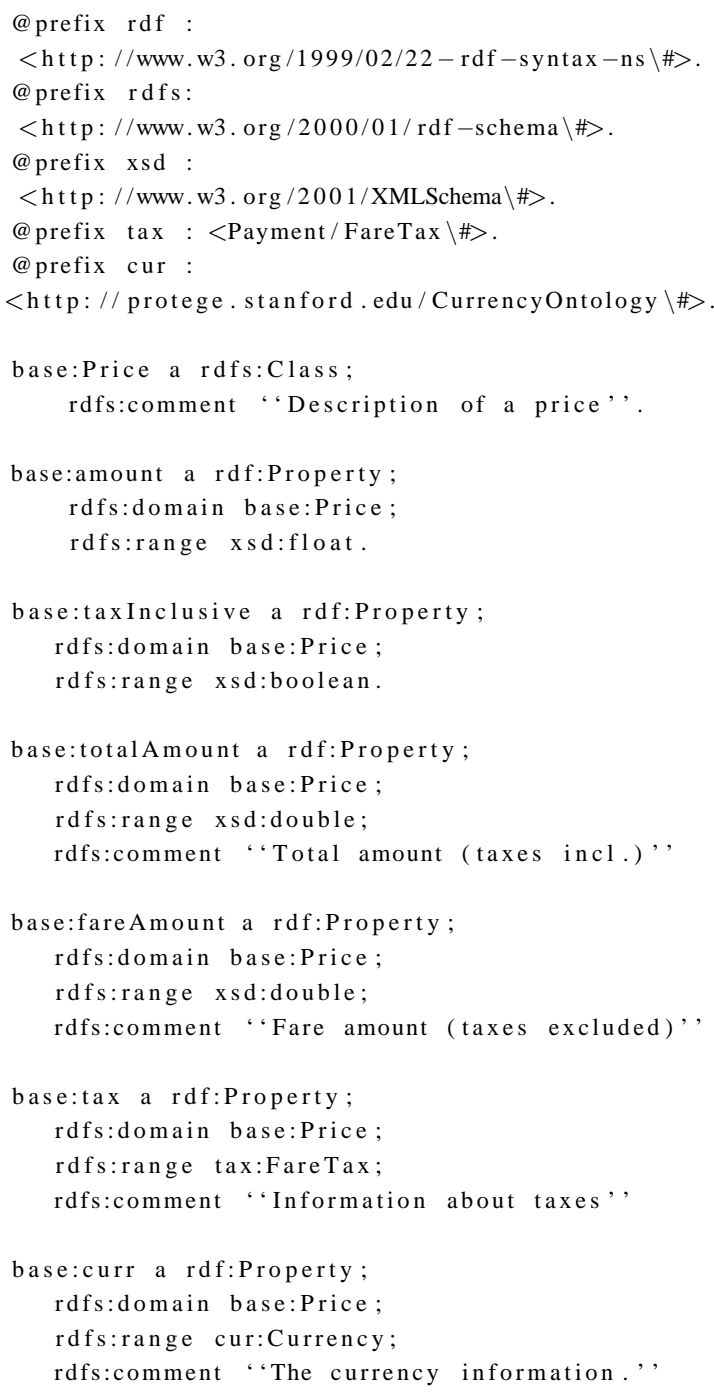

Figure 7: Price concept; proposed Price class

Vukmirovic, M., Szymczak, M., Ganzha, M., and Paprzycki, M. (2006c). Utilizing ontologies in an agentbased airline ticket auctioning system. In et. al., V. L., editor, Proceedings of the 28th ITI Conference, pages 385-390, Piscatawy, NJ. IEEE.

Vukmirovic, M., Szymczak, M., Gawinecki, M., Ganzha, M., and Paprzycki, M. (2007). Designing New Ways for Selling Airline Tickets, volume 31(3), pages 93104 